EXTENDED REPORT

\title{
Course and prognosis of elbow complaints: a cohort study in general practice
}

\section{S D M Bot, J M van der Waal, C B Terwee, D A W M van der Windt, L M Bouter, J Dekker}

See end of article for authors' affiliations

Correspondence to:

Dr C B Terwee, Institute for

Research in Extramural

Medicine (EMGO Institute),

VU University Medical

Centre, Van der

Boechorststraat 7, 1081 BT

Amsterdam;

cb.terwee@vumc.nl

Accepted 2 February 2005

Published Online First

11 February 2005
Objective: To describe the course of new episodes of elbow complaints in general practice, and to identify predictors of short term and long term outcome in terms of pain intensity and functional disability.

Methods: 181 patients with elbow complaints filled in questionnaires at baseline and at 3, 6, and 12 months of follow up. Baseline scores of pain and disability, characteristics of the complaint, sociodemographic and psychosocial factors, physical activity, general health, and comorbidity were investigated as possible predictors of outcome. Outcome measures were analysed separately using multiple regression analyses.

Results: $13 \%$ of the patients reported recovery at the 3 month follow up and $34 \%$ at 12 months. Irrespective of outcome and length of follow up, a longer duration of the complaint before consulting the general practitioner, having musculoskeletal comorbidity, and using "retreating" as coping style increased the likelihood of an unfavourable outcome. Less social support was associated with an unfavourable outcome at 3 months, and having a history of elbow complaints and using "worrying" as coping style were associated with an unfavourable outcome at 12 months. The explained variance of the models ranged from $46 \%$ to $49 \%$.

Conclusions: Recovery of patients with elbow complaints in general practice was poor. Besides characteristic of the complaint, passive coping and less social support were related to a worse prognosis. The results of this study may help general practitioners to provide patients with more accurate information about their prognosis.
$\mathrm{E}$ bow pain and associated disability are common, and affected individuals often consult their general practitioner (GP). Data of the second Dutch national survey of general practice ${ }^{12}$ showed that the incidence of elbow complaints was around 7.2 per 1000 patients per year. ${ }^{3}$ Elbow complaints seldom occur on their own. ${ }^{4}$ Many patients report additional symptoms in the neck, shoulder, arm, or hand. ${ }^{4}$ These complaints can be a considerable burden to both patient and society, owing to inability to work, loss of productivity, and difficulty in carrying out household activities. $^{56}$

Individual characteristics and (work related) physical and psychosocial factors have been identified as risk factors for the onset of elbow complaints. ${ }^{7-9}$ These factors may also act as putative prognostic factors for persistent pain and disability. Not much is known about the prognosis of elbow complaints after presentation in general practice. The vast majority of research on such complaints has been specifically aimed at lateral elbow pain (that is, lateral epicondylitis, tennis elbow, or extensor carpi radialis tendinitis). ${ }^{10}$ Hudak et al reviewed published reports on the clinical course of lateral elbow pain and prognostic factors for outcome. Only four of 40 studies provided at least moderate strength of evidence, showing that the site of the lesion and previous occurrence predicted outcome. ${ }^{10}$ We found only one additional prognostic study of outcome in elbow complaints carried out in general practice, ${ }^{11}$ which showed that high physical strain at work, being employed in manual jobs, high baseline level of distress, a high level of pain at baseline, and a complaint on the dominant side were related to a poor outcome of lateral epicondylitis at one year follow up. So far, little attention has been paid to the potential prognostic value of psychosocial factors, such as coping with pain, kinesiophobia, and social support in general, although these factors were found to be related to a high risk of chronicity in patients consulting their GPs for low back pain ${ }^{12}{ }^{13}$ or neck and shoulder complaints. Knowledge of predictors of outcome should lead to the early identification of those at risk for the development of chronic complaints.

Our objectives in this study were to describe the course of new episodes of elbow complaints in adults in general practice, and to identify predictors associated with short term and long term outcome in terms of pain intensity and functional disability.

\section{METHODS}

\section{Design}

A large observational cohort study was conducted in 61 general practices (97 GPs). ${ }^{14}$ Forty nine of the GPs participated in the second Dutch national survey of general practice (NS2), carried out by the Netherlands Institute of Primary Health Care (NIVEL) in 2001. ${ }^{2}$ GPs recruited patients with a new episode of a complaint at the neck, shoulder, elbow, wrist, or hand. An episode was considered to be "new" if patients had not visited their GP for the same complaint during the preceding three months. Inclusion criteria were age 18 years or older and being capable of filling in Dutch questionnaires. Patients were excluded if the presented symptoms were caused by a fracture, malignancy, prosthesis, amputation, or congenital defect, or if the patient was pregnant. In all, 638 patients (88\%) who consulted their GP with a complaint at the neck or upper extremity complaints

Abbreviations: ACSM, American College of Sports Medicine; MIC, minimum important change; $\mathrm{PCl}$, pain coping inventory; QoL, quality of life; SF-36, 36 item short form health survey 
Table 1 Patient characteristics at baseline $(n=181)$

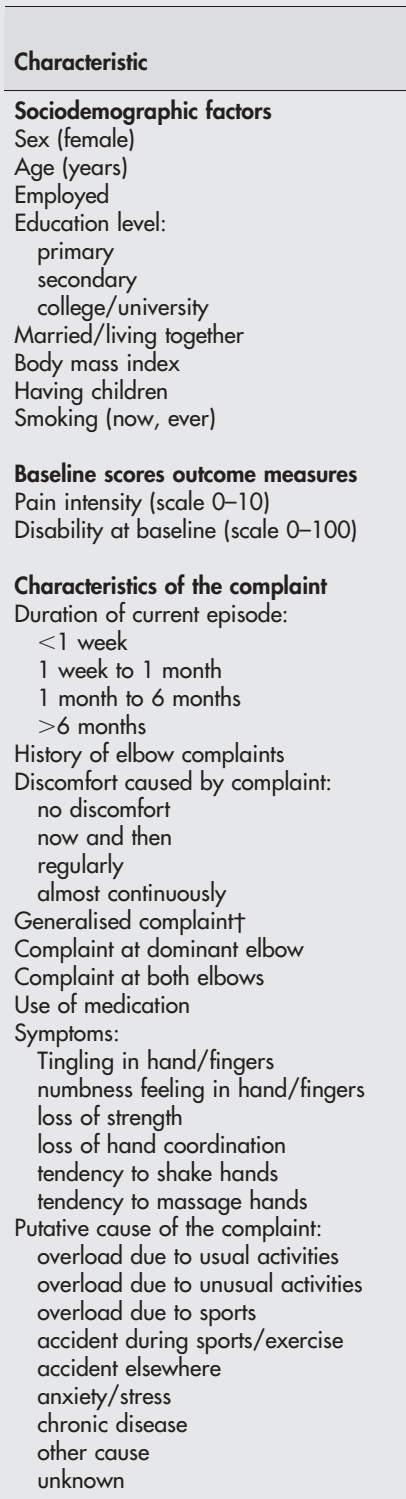

Comorbidity (musculoskeletal)

No comorbidity

Complaints hip/knee

Complaints ankle/foot

Complaints back

Multiple musculoskeletal complaints

Other diseases/problems $\ddagger$

Menopause§

Number of

patients* Per cent

120

$47.9(11.6)$

47.9
112

66

75

84

84
21

138

25.8 (3.9)

88

128

63

41

46

12

76

49

71

$5.3(2.1)$

$34.6(20.4)$

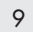

49

49
77

42

97

5

30

57

89

131

98

25

70

79

79
46

46
96

16

43

81

100

23

16

0

14
24

24

25

40

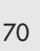

70
42

28

76

29
79

28

Physical activity

ACSM position stand

Norm healthy activity ${ }^{16}$

20

72

\section{Psychosocial factors}

Coping with pain:

$\begin{array}{ll}\text { pain transformation (scale 4-16) } & 8.6(2.6) \\ \text { distraction (scale 5-20) } & 10.8(3.3)\end{array}$

distraction (scale 5-20)

$6.1(2.0)$

$11.1(4.0)$

$16.6(5.0)$

9.6 (3.2)

worrying (scale 9-36)

resting (scale 5-20)

Distress (scale 0-12)

Kinesiophobia:

fear - avoidance (scale 0-100) 52.3 (16.7)

importance of exercise (scale 0-100) 59.9 (20.7)

Social support (scale 12-60)
Table 1 Continued

\begin{tabular}{lll}
\hline Characteristic & $\begin{array}{l}\text { Number of } \\
\text { patients* }^{*}\end{array} \quad$ Per cent \\
\hline General health & $3.0(0.9)$ \\
Perceived health (scale 1-5) & $3.2(0.9)$ \\
Quality of life (scale 1-5) & $57.2(20.2)$ \\
Vitality (scale 0-100) & \\
\hline
\end{tabular}

*Value are mean (SD) for continuous scales or $\mathrm{n}$.

†Patients with concomitant neck, shoulder, arm, hand, or wrist

complaints.

$\ddagger$ Asthma, diabetes, cardiovascular diseases, cancer, psychological

problems.

$\S$ Percentage of women.

-ACSM, American College of Sports Medicine. ${ }^{18}$

as the primary complaint returned the baseline questionnaire. Of these, 181 reported elbow complaints (either as a primary of secondary complaint) and were included in the study. Follow up questionnaires were sent after three, six, and 12 months. ${ }^{14}$

The informed consent procedure and protocol were approved by the medical ethics committee of the VU University Medical Centre. Written informed consent was obtained from all patients.

\section{Outcome measures}

Perceived recovery was measured by asking patients if their complaint still bothered them. Patients that responded "no" were regarded as recovered. Other outcome measures were change in pain intensity and change in functional disability at the three months follow up (short term) and the 12 months follow up (long term). The intensity of the current pain (pain during the previous 24 hours) was measured on an 11 point numerical scale ranging from 0 (no pain) to 10 (unbearable pain). Functional disability was measured with the modified pain free function index, a 10 item elbow specific scale measuring difficulty with the performance of common daily activities, ${ }^{15}$ scores ranging from 0 (not disabled) to 100 (completely disabled). Changes in pain intensity and functional disability were calculated by subtracting the scores at three months and 12 months from the baseline score.

\section{Putative predictors}

We distinguished seven categories of putative predictors measured at baseline:

- Sociodemographic factors: age, sex, body mass index (calculated from self reported weight and height), right/left handedness, marital status, smoking behaviour, and educational background.

- Characteristics of the complaint: duration of the current episode, presumed cause of the complaint, history of elbow complaints, involvement of one or both elbows, complaint at the dominant arm, frequency of discomfort by the complaint, symptoms (for example, tingling in hand/fingers, loss of strength), and the use of analgesics.

- Comorbidity: list of concomitant musculoskeletal complaints, and a list of complaints and diseases other than of the musculoskeletal system.

- Physical activity: We measured whether patients met the norm for healthy activity (yes or no), which recommends that all adults should have 30 minutes or more of moderate intensity physical activity on at least five days of the week, ${ }^{16}{ }^{17}$ and whether they met the American College of Sports Medicine (ACSM) position stand (yes or no), which recommends carrying out heavy physical exercise or sports at least three times a week. ${ }^{18}$ 
- Psychosocial factors: Distress was measured by the shortened version of the distress scale of the four dimensional symptom questionnaire, ${ }^{1920}$ on which a higher score indicates more distress. Coping was measured with the pain coping inventory (PCI), ${ }^{21}{ }^{22}$ consisting of six scales: pain transformation, distraction, reducing demands, retreating, worrying, and resting, a higher score indicating more use of the strategy concerned. Kinesiophobia was measured using two subscales ("fear avoidance beliefs" and "importance of exercise"), derived from the Tampa scale $^{2324}$ and the fear avoidance and beliefs questionnaire, ${ }^{25}$ with a higher score indicating more fear avoidance and finding exercise more important. Social support was measured with the social support scale ${ }^{26}$ on which a higher score indicates less social support.

- General health: vitality was measured by the vitality subscale from the 36 item short form health survey (SF$36)^{27}$; perceived general health was measured with the first question of the general health perceptions subscale of the SF-36 ${ }^{27}$; and perceived overall quality of life (QoL) was measured on a five point rating scale with response options "bad," "moderate," "good," "very good," or "excellent." Higher scores indicate more vitality, better perceived health. and better quality of life.

\section{Statistical analyses}

Univariate regression analyses were undertaken to examine the relation between each of the putative predictors and changes in pain and functioning at the three month and the 12 month follow up. Predictors of recovery could not be studied because of lack of power (only 20 and 51 patients, respectively, were recovered at follow up). Factors that were non-linearly related to the outcome were either dichotomised or divided into tertiles (low, medium, high), with the low category as the reference category. Putative predictors that were associated with the outcome $(p<0.20)$ were

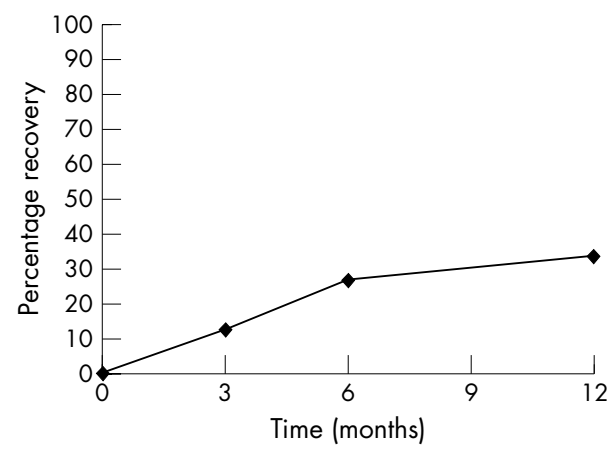

Figure 1 Percentage of recovery in patients with elbow complaints after three, six, and 12 months of follow up.

investigated in a multiple regression model. Age, sex, pain intensity, disability, and duration of the complaint were included in all multiple regression models independent of the $\mathrm{p}$ value. All factors were entered simultaneously in a multiple linear regression model. If the number of putative predictors to be entered in the model exceeded $\mathrm{n} / 10$, the factors were entered in blocks (sociodemographic factors first, characteristics of the complaints next, and the remaining factors last). A manual backward selection procedure was used to sequentially exclude factors and retain only factors with a $p$ value of $<0.10$ (Wald statistic) in the final model, which could be regarded as independent predictors of outcome. The percentage of explained variance $\left(R^{2}\right)$ was calculated to give an indication of the predictive power of the final models.

\section{RESULTS}

Baseline characteristics of the 181 patients are shown in table 1 . The mean (SD) pain intensity score at baseline was 5.3 (2.1) and the mean disability score was 34.6 (20.4); 54\% of the patients reported having had the complaint before in

Table 2 Predictors of change in pain intensity at three months $\left(R^{2}=0.46\right)$ and 12 months $\left(R^{2}=0.47\right)$ : results of the multiple linear regression analyses

\begin{tabular}{|c|c|c|c|c|c|c|c|c|}
\hline & \multicolumn{4}{|c|}{3 Months $(n=157)$} & \multicolumn{4}{|c|}{12 Months $(n=151)$} \\
\hline & $\mathbf{n}$ & $b^{*}$ & $95 \% \mathrm{Cl}$ & p Value & $n$ & $b^{*}$ & $95 \% \mathrm{Cl}$ & $\mathrm{p}$ Value \\
\hline \multicolumn{9}{|l|}{ Demographic factors } \\
\hline Female ( $v$ male) & 107 & -0.94 & $(-1.57$ to -0.31$)$ & 0.00 & & & & \\
\hline older age (per year) & 157 & -0.03 & $(-0.06$ to -0.01$)$ & 0.01 & & & & \\
\hline \multicolumn{9}{|l|}{ Baseline score } \\
\hline More severe pain (per point increase) & 157 & 0.56 & (0.42 to 0.71$)$ & 0.00 & 151 & 0.62 & (0.45 to 0.79$)$ & 0.00 \\
\hline & & & & & & & & \\
\hline \multicolumn{9}{|l|}{$\begin{array}{l}\text { Characteristics of complaint } \\
\text { Duration of current episode: }\end{array}$} \\
\hline$<1$ week & 8 & 0.00 & & & 7 & 0.00 & & \\
\hline 1 week to 1 month & 44 & -1.45 & $(-2.92$ to 0.02$)$ & 0.05 & 42 & -1.63 & $(-3.35$ to 0.09$)$ & 0.06 \\
\hline 1 month to 6 months & 66 & -1.76 & $(-3.21$ to -0.30$)$ & 0.02 & 64 & -1.63 & $(-3.32$ to 0.05$)$ & 0.06 \\
\hline$>6$ months & 36 & -2.19 & $(-3.70$ to -0.68$)$ & 0.00 & 36 & -2.36 & $(-4.14$ to -0.59$)$ & 0.01 \\
\hline History of complaints ( $v$ no history) & & & & & 83 & -1.50 & $(-2.27$ to -0.74$)$ & 0.00 \\
\hline Complaint at dominant side ( $v$ other side) & 86 & 0.82 & $(0.22$ to 1.41$)$ & 0.01 & & & & \\
\hline Tendency to massage hands ( $v$ no tendency) & & & & & 66 & -0.88 & $(-1.55$ to -0.21$)$ & 0.01 \\
\hline \multicolumn{9}{|l|}{ Comorbidity } \\
\hline No musculoskeletal comorbidity ( $v$ comorbidity) & 63 & 0.55 & $(-0.09$ to 1.19$)$ & 0.09 & & & & \\
\hline $\begin{array}{l}\text { Multiple musculoskeletal complaints ( } \mathrm{v} \text { "no") } \\
\text { Psychosocial factors }\end{array}$ & 24 & -0.98 & $(-1.83$ to -0.12$)$ & 0.03 & 25 & -1.23 & $(-2.14$ to -0.31$)$ & 0.01 \\
\hline Coping: more retreating (per point increase) & 155 & -0.09 & $(-0.17$ to -0.01$)$ & 0.03 & 150 & -0.10 & $(-0.20$ to -0.01$)$ & 0.04 \\
\hline Coping: more worrying (per point increase) & & & & & 150 & -0.10 & $(-0.18$ to -0.02$)$ & 0.01 \\
\hline More fear voidance (per point increase) & 156 & 0.02 & $(0.00$ to 0.04$)$ & 0.05 & & & & \\
\hline \multicolumn{9}{|l|}{ Social supportt } \\
\hline low & 51 & 0.00 & & & & & & \\
\hline medium & 69 & -0.70 & $(-1.37$ to -0.02$)$ & 0.04 & & & & \\
\hline high & 35 & -1.09 & $(-1.92$ to -0.27$)$ & 0.01 & & & & \\
\hline
\end{tabular}


the previous year (that is, there was a history of the complaint).

In all, 158 patients (87\%) completed the three months follow up questionnaire, and 152 patients (84\%) completed the 12 month questionnaire. There were no significant differences between responders and dropouts in age, sex, functional disability, and pain intensity.

\section{Course}

After three months, 20 patients (13\%) reported recovery, and 51 patients (34\%) reported recovery after 12 months (fig 1 ). Of the patients who were not fully recovered at three months, $24 \%$ reported substantial improvement and 37\% reported some improvement compared with baseline. At 12 months, $21 \%$ of patients without full recovery reported substantial improvement and $25 \%$ some improvement. At follow up the mean (SD) reduction in pain intensity was $1.3(2.3)$ points at three months and $2.1(2.6)$ points at 12 months $(\mathrm{p}<0.01)$.

The mean reduction in disability was $6.3(16.2)$ points at three months and $11.9(21.2)$ points at 12 months $(\mathrm{p}<0.01)$.

\section{Predictors of outcome: univariate analysis}

The results of the univariate regression analyses showing the associations between the putative predictors and change in pain intensity and change in functional disability after three or 12 months are presented in the appendix (the appendix can be viewed on the journal website at www.annrheumdis. com/supplemental). Age, a history of elbow complaints, having additional musculoskeletal complaints, using the coping strategy "retreating", and social support were associated with both outcomes at both follow up periods.

\section{Predictors of outcome: pain}

More intense pain at baseline, having a complaint in the dominant arm, and a higher score on the fear avoidance scale were significantly associated with a better outcome at three months (table 2). A worse outcome at three months was predicted by being female, higher age, a longer duration of the complaint at presentation, having multiple additional musculoskeletal complaints, using retreating as a coping strategy, and having less social support. For example, the improvement in pain intensity at three months for a women $(-0.94)$ who had the complaint more than six months $(-2.19)$ was 3.13 points less than for a man who had the complaint for less than one week, provided that the other predictors of the model were similar.

Predictors of a poorer outcome at 12 months were less pain at baseline, a longer duration of the complaint at presentation, having had the complaint before, having a tendency to massage your hands, having multiple musculoskeletal complaints, and scoring high on retreating and worrying (table 2). The explained variance was 0.46 at the three months follow up and 0.47 at 12 months.

\section{Predictors of outcome: functional disability}

Being employed, being more disabled at baseline, and having an accident as the presumed cause of the complaint were independently associated with a better outcome at three months (table 3 ). Factors that predicted a worse outcome were: having children in the household, more intense pain at baseline, a longer duration of the complaint at presentation, having multiple musculoskeletal complaints, a high score on the pain coping scale "retreating," and less social support.

Being less disabled at baseline, having more intense pain at baseline, a longer duration of the complaint at presentation, chronic disease as the presumed cause of the complaint, additional complaints at the hip or knee, and a higher score on the pain coping scales "retreating" and "worrying" were significantly associated with a worse outcome at 12 months (table 3). The explained variance of the models for change in functional disability was 0.49 at three months and 0.47 at 12 months.

Table 3 Predictors of change in disability at three months $\left(R^{2}=0.49\right)$ and 12 months $\left(R^{2}=0.47\right)$ : results of the multiple linear regression analyses

\begin{tabular}{|c|c|c|c|c|c|c|c|c|}
\hline & \multicolumn{4}{|c|}{3 Months ( $n=157$ ) } & \multicolumn{4}{|c|}{12 Months $(n=151)$} \\
\hline & $\mathbf{n}$ & $b^{*}$ & $95 \% \mathrm{Cl}$ & $\mathrm{p}$ Value & $\mathbf{n}$ & $\boldsymbol{b}^{*}$ & $95 \% \mathrm{Cl}$ & $\mathrm{p}$ Value \\
\hline \multicolumn{9}{|l|}{ Sociodemographic factors } \\
\hline Employed ( $v$ unemployed) & 95 & 5.43 & (1.26 to 9.61$)$ & 0.01 & & & & \\
\hline No children ( $v$ having children) & 80 & 4.96 & (0.98 to 8.93$)$ & 0.01 & & & & \\
\hline \multicolumn{9}{|l|}{ Baseline score } \\
\hline More severe pain (per point increase) & 157 & -1.88 & $(-3.13$ to -0.63$)$ & 0.00 & 150 & -1.37 & $(-2.99$ to 0.25$)$ & 0.10 \\
\hline More disabled (per point increase) & 156 & 0.58 & (0.45 to 0.72$)$ & 0.00 & 151 & 0.71 & (0.54 to 0.87$)$ & 0.00 \\
\hline \multicolumn{9}{|l|}{ Characteristics of complaint } \\
\hline \multicolumn{9}{|l|}{ Duration of current episode: } \\
\hline$<1$ week & 8 & 0.00 & & & 7 & 0.00 & & \\
\hline 1 week to 1 month & 44 & -8.67 & $(-19.40$ to 2.06$)$ & 0.11 & 42 & -10.10 & $(-23.94$ to 3.73$)$ & 0.15 \\
\hline 1 month to 6 months & 66 & -9.10 & $(-19.44$ to 1.25$)$ & 0.08 & 64 & -11.30 & $(-24.87$ to 2.27$)$ & 0.10 \\
\hline$>6$ months & 36 & -12.18 & $(-23.07$ to -1.28$)$ & 0.03 & 36 & -14.84 & $(-29.14$ to -0.53$)$ & 0.04 \\
\hline History of complaints ( $v$ no history) & & & & & 83 & -9.77 & $(-15.97$ to -3.57$)$ & 0.00 \\
\hline Putative cause: accident ( $v$ "no") & 13 & 8.58 & (1.09 to 16.07$)$ & 0.03 & & & & \\
\hline Putative cause: chronic disease ( $\left.v^{\prime \prime} n \mathrm{o}^{\prime \prime}\right)$ & & & & & 10 & -10.07 & $(-21.27$ to 1.12$)$ & 0.08 \\
\hline \multicolumn{9}{|l|}{ Comorbidity } \\
\hline Complaints at hip/knee ( $v$ "no") & & & & & 37 & -9.21 & $(-16.04$ to -2.38$)$ & 0.01 \\
\hline $\begin{array}{l}\text { Multiple musculoskeletal complaints ( } v \text { "no") } \\
\text { Psychosocial factors }\end{array}$ & 24 & -9.66 & $(-15.56$ to -3.77$)$ & 0.00 & & & & \\
\hline Coping: more retreating (per point increase) & 155 & -0.88 & $(-1.42$ to -0.34$)$ & 0.00 & 150 & -0.92 & $(-1.72$ to -0.12$)$ & 0.03 \\
\hline Coping: worrying (per point increase) & & & & & 150 & -0.68 & $(-1.33$ to -0.03$)$ & 0.04 \\
\hline \multicolumn{9}{|l|}{ Social supportt } \\
\hline low & 51 & 0.00 & & & & & & \\
\hline medium & 69 & -4.69 & $(-9.38$ to 0.01$)$ & 0.05 & & & & \\
\hline high & 35 & -2.78 & $(-8.50$ to 2.94$)$ & 0.34 & & & & \\
\hline
\end{tabular}




\section{DISCUSSION}

We have described the course of elbow complaints in general practice and examined potential predictors of outcome. Only $13 \%$ of reported full recovery after three months and just one third of the patients recovered after one year of follow up. These figures are consistent with those of Hay et al, who reported a low overall recovery rate ( $17 \%$ ) after four weeks in primary care patients with lateral epicondylitis. ${ }^{28}$ Other studies have used general improvement as outcome measures instead of complete recovery and have found percentages of $69-85 \%$ after one year. ${ }^{11}{ }^{29}$ In our study $90 \%$ of all patients reported at least some improvement after one year of follow up. The mean reduction in pain intensity was $1.3(2.3)$ points at three months and 2.1 (2.6) points at 12 months. In clinical practice this may be considered to be meaningful: research has shown that a reduction of one point on a $0-10$ point scale represents the minimum clinically important change in pain intensity (MIC). ${ }^{3031}$ The mean reduction in disability score was 6.3 (16.2) points at three months and $11.9(21.2)$ points at 12 months. The MIC of this functional disability scale is unknown. However, in most circumstances the MIC appears to be approximately half a standard deviation. ${ }^{32}$ In this case, half a standard deviation would be approximately 10 points, which means that the observed changes in functional disability were only clinically important after 12 months of follow up.

Irrespective of outcome measure and length of follow up, a worse prognosis was found for patients who had a longer duration of their complaint at baseline. This is in agreement with studies on the prognosis of musculoskeletal complaints in the neck or shoulder. ${ }^{33-35}$ Not surprisingly pain intensity and functional disability at baseline were strongly associated with changes in these outcomes at follow up. Having more pain or disability at baseline leaves more room for a large reduction at follow up, but does not necessarily result in a better prognosis, as these patients may still have considerable pain or disability at follow up. For instance, a patient with a baseline pain score of 9 and a follow up score of 6 improved more than a patient with a baseline score of 3 and a follow up score of 1. This may also explain why a higher pain score at baseline predicted a poorer functional disability at follow up. Having had elbow complaints in the past predicted a worse outcome at long term follow up. This is similar to the results of Hudak et al. ${ }^{10}$

The passive coping styles retreating (for example, "make sure that I don't get upset"; "separate myself") and worrying ("focus on pain all the time"; "I think the pain will get worse") independently predicted poorer outcome. Passive coping strategies are thought to generate a preoccupation with bodily symptoms, which in turn may increase the sensation of pain and disability. ${ }^{36}{ }^{37}$ Several studies have found that a passive coping strategy is associated with a poor outcome in neck and back pain, ${ }^{12} 38$ and in patients with rheumatoid arthritis. ${ }^{36} 3940$

Less social support was associated with worse short term outcome in our study. Low levels of social support at the time of diagnosis predicted pain and functional disability in patients with rheumatoid arthritis at long term follow up. ${ }^{37}$ Social resources may affect the health status of patients by enhancing the ability of an individual to cope with the stress of their disease.

In contrast to the results of Haahr and Anderson, ${ }^{11}$ we found an association between sex and age and pain at three months follow up. Furthermore, they found that lateral epicondylitis at the dominant side predicted a worse prognosis at 12 months, while in our study it predicted a favourable outcome at three months. As well as a difference in study population (we studied patients with a variety of elbow complaints), it is possible that the difference in the follow up period may explain these contrasting findings.
We may have missed some predictors of short and long term elbow complaints or that we may have found some associations by chance. Treatment could be an important predictor of outcome. ${ }^{29}$ In the study by Hay et al the recovery rate after four weeks differed substantially between treatment groups $(4 \%$ to $42 \%) .^{28}$ In everyday clinical practice decisions to treat are often dependent on indicators of prognosis, such as the duration or severity of symptoms. Thus differences in outcome are not only related to the treatment given but also to differences in the severity of the condition. Consequently, interpretation of the predictive value of treatment variables is very difficult in observational research, as this information cannot be interpreted as evidence for the effectiveness of treatment. We therefore decided not to include treatment variables in our prognostic model. In addition, we did not included work related factors, because more than one third of the study population did not have paid work.

Lateral epicondylitis is the most common problem among patients with elbow complaints. The Dutch general practice guidelines recommend a wait and see policy for epicondylitis; injections or physiotherapy are only recommended in patients with persistent and severe pain or disability. ${ }^{42}$ Although we have no information how well GPs adhere to these guidelines, we assume that in most cases the initial treatment will be based on an expectant policy, which includes time limited prescription of drugs and recommendations for temporarily avoiding pain provoking activities. Our results offer additional evidence on the prognostic value of coping and social support, which may be relevant to future updates of the guidelines. However, owing to the observational design of our study, our results provide only preliminary evidence for a causal association between the predictors found and changes in pain and functional disability in elbow complaints. The predictive capability of the model should be assessed in another population of patients with elbow complaints.

Our study suggests that few patients who consult their GP with a new episode of an elbow complaint have full recovery of their symptoms, though $90 \%$ of all patients reported at least some improvement after one year of follow up. The average pain and disability scores diminished after three and 12 months, although the improvement in disability can only be considered important after 12 months. Several factors were found to be independent predictors of outcome. As well as the characteristic of the complaint (for example, duration, history of complaints), passive coping and less social support were related to a worse prognosis. The results of our study may help GPs to identify patients at risk for the development of chronic complaints and provide them with more accurate information on their prognosis. More high quality studies in general practice are needed to confirm our results.

\section{ACKNOWLEDGEMENTS}

This study is financially supported by the Dutch Arthritis Association.

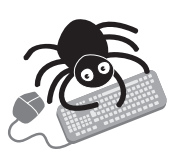

The electronic supplementary appendix can be found on the journal web site, www.annrheumdis.com/ supplemental

\section{Authors' affiliations}

S D M Bot, J M van der Waal, C B Terwee, L M Bouter, Institute for Research in Extramural Medicine, VU University Medical Centre, Amsterdam, Netherlands

D A W M van der Windt, Department of General Practice, VU University Medical Centre

J Dekker, Department of Rehabilitation Medicine, VU University Medical Centre 


\section{REFERENCES}

1 Schellevis FG, Westert GP, de Bakker DH, Groenewegen PP, van der Zee J, Bensing JM. De tweede nationale studie naar ziekten en verrichtingen in de huisartsenpraktijk: aanleiding en methoden. Huisarts en Wetenschap 2003:46:7-12

2 Westert GP, Schellevis FG, de Bakker DH, Groenewegen PP, Bensing JM, van der Zee J. Monitoring health inequalities through general practice: the Second Dutch National Survey of General Practice. Eur J Public Health 2004; 15:59-65

3 Bot SD, van der Waal JM, Terwee CB, van der Windt DA, Schellevis FG, Bouter $L M$, et al. Incidence and prevalence of complaints at the neck and upper extremity in general practice. Ann Rheum Dis 2005;64:118-23.

4 Walker-Bone K, Reading I, Coggon D, Cooper C, Palmer KT. The anatomical pattern and determinants of pain in the neck and upper limbs: an epidemiologic study. Pain 2004;109:45-51.

5 Reville RT, Neuhauser FW, Bhattacharya J, Martin C. Comparing severity of impairment for different permanent upper extremity musculoskeletal injuries. J Occup Rehabil 2002;12:205-221.

6 Williams R, Westmorland M. Occupational cumulative trauma disorders of the upper extremity. Am J Occup Ther 1994;48:411-20.

7 Bernard BP. Musculoskeletal disorders and workplace factors: a critical review of epidemiologic evidence for work-related musculoskeletal disorders of the neck, upper extremity, and low back. Publication No 97-141. Cincinnati: National Institute for Occupational Safety and Health, 1997.

8 Haahr JP, Andersen JH. Physical and psychosocial risk factors for lateral epicondylitis: a population based case-referent study. Occup Environ Med 2003;60:322-9

9 Walker-Bone KE, Palmer KT, Reading I, Cooper C. Soft-tissue rheumatic disorders of the neck and upper limb: prevalence and risk factors. Semin Arthritis Rheum 2003;33:185-203.

10 Hudak PL, Cole DC, Haines AT. Understanding prognosis to improve rehabilitation: the example of lateral elbow pain. Arch Phys Med Rehabil 1996:77:586-93.

11 Haahr JP, Andersen JH. Prognostic factors in lateral epicondylitis: a randomized trial with one-year follow-up in 266 new cases treated with minimal occupational intervention or the usual approach in general practice. Rheumatology (Oxford) 2003:42:1216-25.

12 Burton AK, Tillotson KM, Main CJ, Hollis S. Psychosocial predictors of outcome in acute and subchronic low back trouble. Spine 1995;20:722-8.

13 Klenerman L, Slade PD, Stanley IM, Pennie B, Reilly JP, Atchison LE, et al. The prediction of chronicity in patients with an acute attack of low back pain in a general practice setting. Spine 1995;20:478-84.

14 van der Waal JM, Bot SD, Terwee CB, van der Windt DA, Bouter LM, Dekker J. Determinants of the clinical course of musculoskeletal complaints in general practice: design of a cohort study. BMC Musculoskel Disord 2003;4:3.

15 Stratford P, Levy D, Levy K, Miseferi D. Extensor carpi radialis tendonitis: a validation of selected outcome measures. Physiotherapy Can 1987;39:250-5.

16 Kemper H, Ooijendijk W, Stiggelbout M. Consensus about the Dutch recommendation for physical activity to promote health (Consensus over de Nederlandse norm gezond bewegen). Tiidschriff voor Gezondheidswetenschappen 2000;78:180-3.

17 Pate RR, Pratt M, Blair SN, Haskell WL, Macera CA, Bouchard C, et al. Physical activity and public health. A recommendation from the Centers for Disease Control and Prevention and the American College of Sports Medicine. JAMA 1995;273:402-7.

18 American College of Sports Medicine Position Stand. the recommended quantity and quality of exercise for the developing and maintaining cardiorespiratory and muscular fitness in healthy adults. Med Sci Sports Exerc 1990;22:265-74

19 Terluin B. The Four Dimensional Symptom Questionnaire (4DSQ) in general practice. De Psycholoog 1998;33:18-24.

20 Terluin B, van Rhenen W, Schaufeli WB, de Haan M. The four-dimensional symptom questionnaire (4SDQ): measuring distress and other mental health problems in a working population. Work Stress 2004;18:187-207.
21 Kraaimaat FW, Evers AW. Pain-coping strategies in chronic pain patients: psychometric characteristics of the pain-coping inventory (PCI). Int J Behav Med 2003; 10:343-63.

22 Kraaimaat $F$, Bakker A, Evers A. Pain coping strategies in chronic pain patients: the development of the Pain Coping Inventory (PCI). Gedragstherapie 1997;30:185-201.

23 Goubert L, Crombez G, Vlaeyen J, an Damme S, van den Broeck A, van den Houdenhove B. The Tampa Scale for Kinesiophobia: Psychometric characteristics and norms. Gedrag en Gezondheid 2000;28:54-62.

24 Goubert L, Crombez G, Van Damme S, Vlaeyen JW, Bijttebier P, Roelofs J. Confirmatory factor analysis of the Tampa Scale for Kinesiophobia: invariant two-factor model across low back pain patients and fibromyalgia patients. Clin J Pain 2004;20:103-10.

25 Waddell G, Newton M, Henderson I, Somerville D, Main CJ. A FearAvoidance Beliefs Questionnaire (FABQ) and the role of fear-avoidance beliefs in chronic low back pain and disability. Pain 1993;52:157-68.

26 Feij J, Doorn C, van Kampen D, van den Berg P, Resing W. Sensation seeking and social support as moderators of the relationship between life events and physical illness/psychological distress. In: Winnubst JAM, Maes S, eds. Lifestyles stress and health. Leiden: DSWO Press, 1992:285-302.

27 Ware JE, Sherbourne CD. The MOS 36-item short-form health survey (SF-36). I. Conceptual framework and item selection. Med Care 1992;30:473-83.

28 Hay EM, Paterson SM, Lewis M, Hosie G, Croft P. Pragmatic randomised controlled trial of local corticosteroid injection and naproxen for treatment of lateral epicondylitis of elbow in primary care. BMJ 1999;319:964-8.

29 Smidt N, van der Windt DA, Assendelft WJ, Deville WL, Korthals-de Bos IB, Bouter LM. Corticosteroid injections, physiotherapy, or a wait-and-see policy for lateral epicondylitis: a randomised controlled trial. Lancet 2002;359:657-62.

30 Cepeda MS, Africano JM, Polo R, Alcala R, Carr DB. What decline in pain intensity is meaningful to patients with acute pain? Pain 2003;105:151-7.

31 Salaffi F, Stancati A, Alberto SC, Ciapetti A, Grassi W. Minimal clinically important changes in chronic musculoskeletal pain intensity measured on a numerical rating scale. Eur J Pain 2004;8:283-91.

32 Norman GR, Sloan JA, Wyrwich KW. Interpretation of changes in healthrelated quality of life: the remarkable universality of half a standard deviation. Med Care 2003:41:582-92.

33 Hoving JL, de Vet HC, Twisk JW, Deville WL, van der Windt D, Koes BW, et al. Prognostic factors for neck pain in general practice. Pain 2004; 110:639-45.

34 Kjellman G, Skargren E, Oberg B. Prognostic factors for perceived pain and function at one-year follow-up in primary care patients with neck pain. Disabil Rehabil 2002;24:364-70

35 Kuijpers T, van der Windt DA, van der Heijden GJ, Bouter LM. Systematic review of prognostic cohort studies on shoulder disorders. Pain 2004; 109:420-31.

36 Covic T, Adamson B, Hough M. The impact of passive coping on rheumatoid arthritis pain. Rheumatology (Oxford) 2000;39:1027-30.

37 Evers AW, Kraaimaat FW, Geenen R, Jacobs JW, Biilsma JW. Pain coping and social support as predictors of long-term functional disability and pain in early rheumatoid arthritis. Behav Res Ther 2003:41:1295-310.

38 Linton SJ. Occupational psychological factors increase the risk for back pain: a systematic review. J Occup Rehabil 2001;11:53-66.

39 Evers AW, Kraaimaat FW, Geenen R, Bijlsma JW. Psychosocial predictors of functional change in recently diagnosed rheumatoid arthritis patients. Behav Res Ther 1998;36:179-93.

40 Smith CA, Wallston KA, Dwyer KA, Dowdy SW. Beyond good and bad coping: a multidimensional examination of coping with pain in persons with rheumatoid arthritis. Ann Behav Med 1997;19:11-21.

41 Waltz M, Kriegel W, van't Pad BP. The social environment and health in rheumatoid arthritis: marital quality predicts individual variability in pain severity. Arthritis Care Res 1998;11:356-74

42 Assendelft WJ, Rikken SA, Mel M, Schoonheim PL, Schoenmaker PK, Dijkstra HR, et al. NHG-standaard Epicondylitis. Huisarts en Wetenschap 1997;40:21-6. 